\title{
Application of Hyperspectral Data for Land Cover Classification
}

\author{
Amarsaikhan Damdinsuren ${ }^{1}$, Byambadolgor Batdorj ${ }^{1, *}$, Jargaldalai Enkhtuya $^{1}$, \\ Enkhjargal Damdinsuren ${ }^{1}$, Tsogzol Gurjav ${ }^{1}$ \\ ${ }^{1}$ Institute of Geography and Geoecology, Mongolian Academy of Sciences, Ulaanbaatar, Mongolia \\ *Corresponding author. Email: byambadolgorb@mas.ac.mn
}

\begin{abstract}
At present, hyperspectral imaging techniques are widely used for a variety of different thematic applications, because they record a detailed spectrum of incoming radiation for every pixel and provide an invaluable source of information related to the physical nature of the Earth's surface features. Generating accurate land cover maps using remote sensing (RS) datasets is one of the most important applications of digital image processing. For the generation of accurate maps, diverse supervised, unsupervised and hybrid classification methods can be applied. As hyperspectral images contain abundant spectral information, it makes them possible to distinguish various objects that would not be distinguishable by multispectral sensors. The aim of this study is to discriminate the land cover types in northern Mongolia using some advanced hyperspectral image classification techniques. As data sources, a Hyperion image of 2014 and some other ground truth information have been used. Overall, the research indicated that modern advanced hyperspectral data analysis methods could be successfully used for the land cover classification.
\end{abstract}

Keywords: Hyperspectral image, advanced classification, land cover

\section{INTRODUCTION}

Unlike the traditional multispectral sensors, the hyperspectral sensors acquire images with hundreds of narrow spectral bands within the visible, near infrared, middle infrared ranges of the electromagnetic spectrum. Hyperspectral imaging systems can make it possible to extract some appearance features that are difficult or impossible with the traditional computer vision systems. The goal of hyperspectral imaging is to obtain the spectrum for each pixel in the image of a scene, with the purpose of finding objects, identifying materials, or detecting processes. To obtain high spectral resolution and narrow band image data, hyperspectral imaging is generally combined with spectroscopic technique, two-dimensional geometric space and onedimensional spectral information detection. Therefore, with the rapid development of information science, digital image processing and pattern recognition technology, optical sensing technologies have been emerged as advanced tools for evaluation of different land cover and use types. Spectral imaging technology, combining conventional imaging and spectroscopy techniques, can acquire accurate spatial and spectral information from the Earth's objects [1].

In RS data analysis, image classification plays an important role in extracting thematic information from the primary digital dataset. Traditional classification methods mainly include supervised and unsupervised techniques [2]. In the supervised classification one should select sample pixels in an image that are representative of specific classes and then direct the image processing algorithm to use these training signatures as references for the classification of other pixels in the image. In the unsupervised classification, the algorithm automatically groups pixels with similar spectral characteristics into unique clusters on the basis of some statistically determined conditions. Since hyperspectral image classification deals with a problem with high-dimensional feature space and low number of labeled data, the majority of the common algorithms suffer a lot from curse of dimensionality [3]. To tackle this problem, researchers from the pattern recognition community try to introduce 
variety of advanced classification techniques and feature extraction methods.

As hyperspectral imaging records a detailed spectrum of light for each pixel, it provides an invaluable source of information regarding the physical nature of the different features, leading to the potential of a more accurate classification. That is abundant spectral information in hyperspectral datasets makes it possible to distinguish the objects of classes that could not be distinguishable by multispectral sensors [4]. However, hyperspectral image classification still has been recognized as the fundamental and interesting task in the field of digital RS data processing because of the Hughes phenomenon. The Hughes phenomenon deals with an increase in dimensions of limited training samples that cause a decrease in classification performance. To solve this problem, the scholars are developing various techniques in hyperspectral image analysis, but due to the spatial variability of spectral signatures, most of the efforts faced different challenges [5].

In recent years, in the fields of hyperspectral image processing different advanced classification techniques have been developed. Many of these methods can overcome the above mentioned Hughes phenomenon, if a sufficient number of training samples are selected. Within the framework of this research, we wanted to discriminate land cover types in northern Mongolia using some advanced methods applied for the hyperspectral image classification. As data sources, 242 band Hyperion image of 25 June 2014 and some other ground truth information have been used.

\section{TEST AREA AND DATA SOURCES}

In this Mongolia has relatively low forest cover with just over 8 percent of the country covered by closed forests. The forests are mainly located in the north-central parts of the country, forming a transition zone between the Siberian boreal forest and the Central Asian steppe desert. In northern Mongolia, the mountain slopes are clothed with boreal taiga forest. Due to a brief warm period, the growing season is not long enough for many plant species. It forms the most southern extension of the east Siberian taiga and includes mainly larch and pine and rich in mosses and lichens. Mongolian forests also provide a multitude of important functions with respect to climate change and other environmental problems, carbon sinks, watershed protection, playing important role in preservation of permafrost in its ecologically important form and reduction of harmful emissions [6].

As a test site, a coniferous forest-dominated area situated in western part of the Lake Khuvsgul, northern Mongolia has been selected. The lake is considered as the second largest fresh water lake in Asia after the Lake Baikal with $100 \mathrm{~km}$ in length, 35 $\mathrm{km}$ in width, and over $265 \mathrm{~m}$ in depth. The lake lies at $1645 \mathrm{~m}$ above sea level where mountains on the western shore rise up to $2961 \mathrm{~m}$ and the mountains on the northern shore even up to $3491 \mathrm{~m}$. The annual precipitation in the region is about $350-400 \mathrm{~mm}$ and it makes the area as the most humid region in the country. The area selected in the west of the Lake Khuvsgul represents a forest ecosystem and is characterized by such main classes as coniferous forest, grassland, soil, bare land, dry river bed and water.

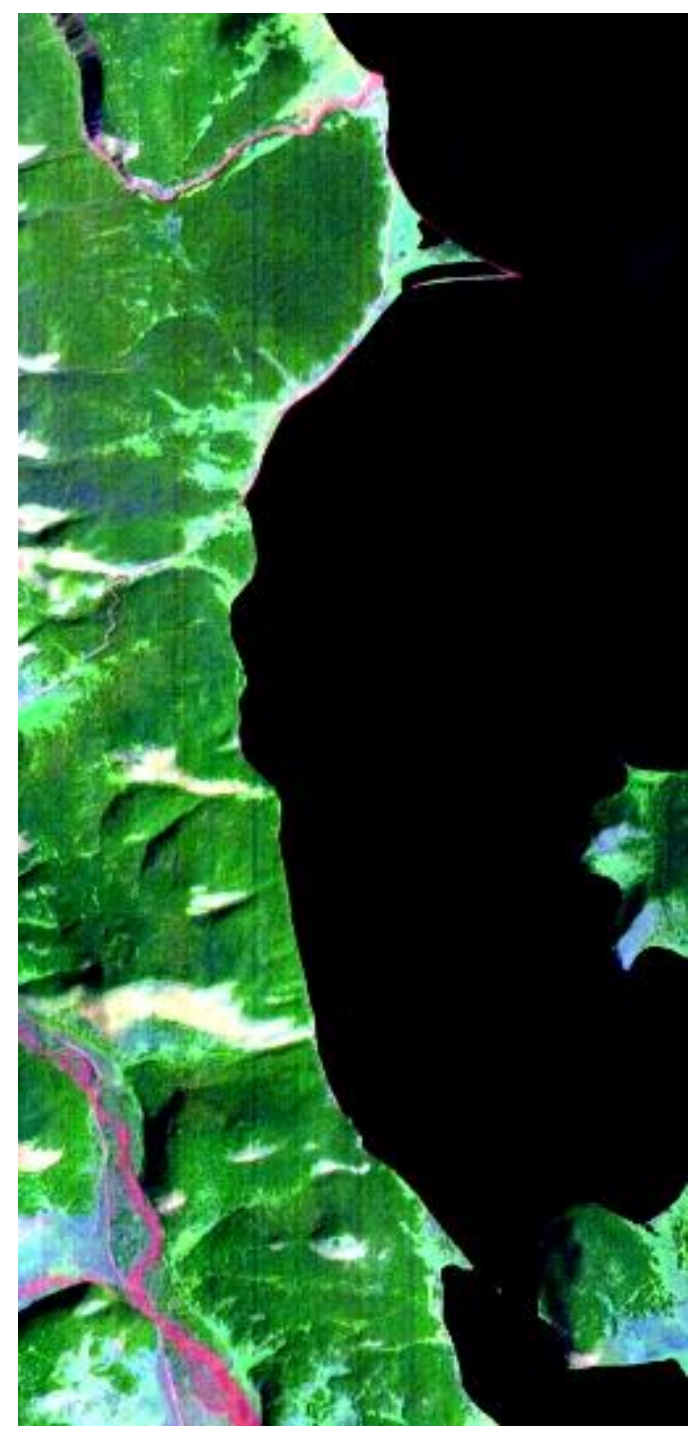

Figure 1. Hyperion image of the test area 
As RS data, hyperspectral Hyperion image of 25 June 2014 has been used. The Hyperion is a high resolution hyperspectral imaging instrument that was carried out on EO-1 mission. It is the first satellite in NASA's New Millennium Program Earth Observing series and can image the Earth's surface in 242 contiguous spectral bands with high radiometric accuracy, covering the region from $0.4 \mu \mathrm{m}$ to $2.5 \mu \mathrm{m}$, at a ground resolution of $30 \mathrm{~m}$. Through this large number of spectral bands, complex land and water surface features can be accurately imaged [7]. In addition, a topographic map of scale 1:100,000 and a forest taxonomy map of 1984, scale 1: 500,000 were available. The selected test site in the Hyperion image frame is shown in Figure 1.

\section{CLASSIFICATION METHODS}

Spectral angle mapper determines the spectral similarity between two spectra by calculating the angle between the spectra and treating them as vectors in space with dimensionality equal to the number of bands. This technique, when used on calibrated reflectance data, is relatively insensitive to illumination and albedo effects. End-member spectra used by spectral angle mapper can come from spectral libraries, or one can extract them directly from an image as a signature. The method compares the angle between the end-member spectrum vector and each pixel vector in $n-D$ space. Smaller angles represent closer matches to the reference spectrum. Pixels positioned distant away than the specified maximum angle threshold in radians are not classified. The weakness of the method is that it is insensitive to the unknown gain factor. All possible illuminations are treated equally. Poorly illuminated pixels fall closer to the origin of the scatter plot. The color of a material is defined by the direction of its unit vector. The angle between the vectors is the same, regardless of the length. The length of the vector relates only to how fully the pixel is illuminated [8].

Support vector machine is a linear model for classification problems. It can solve linear and nonlinear problems and work well for many practical problems. This is one of the most vigorous prediction methods based on statistical learning frameworks. Given a set of training examples, each marked as belonging to one of two categories, a training algorithm builds a model that assigns new examples to one category or the other, making it a nonprobabilistic binary classifier. Support vector machine maps training examples to points in space so as to maximize the width of the gap between the two categories. New examples are then mapped into that same space and predicted to belong to a category based on which side of the gap they fall. More formally, the method constructs a set of hyper-planes in a high or infinite dimensional space, which can be used for decision-making. An acceptable separation is achieved by the hyper-plane that has the largest distance to the nearest training-data point of any class, since in general the larger the margin, the lower the error of the classifier [9].

Spectral information divergence is a spectral classification method that uses a divergence measure to match pixels to reference spectra. The smaller the divergence, the more likely the pixels are similar. Pixels with a measurement greater than the specified maximum divergence threshold are not classified. Unlike the spectral angle mapper method which calculates the spectral angle between two spectra, each pixel spectrum in the spectral information divergence is considered as a random variable and the divergence of probabilistic behaviors between the two spectral vectors is measured. In other words, the method views each pixel spectrum as a random variable and then measures the discrepancy of probabilistic behaviors between two spectra. The end-member spectra used by the method usually come from spectral libraries, or one can extract them directly from an image as a signature $[10 ; 11]$.

Binary spectral encoding encodes the data and end-member spectra into zeros and ones, based on whether data falls below or above the spectrum mean, correspondingly. Many consider it as a procedure to convert data to a form easily used by computer operating systems and achieved by converting binary data to a string format, specifically, converting 8-bit data into other acceptable bit formats. An exclusive OR function compares each encoded reference spectrum with the encoded data spectra and produces a classified image. All pixels are classified to the endmember with the greatest number of bands that match, unless you specify a minimum match threshold, in which case some pixels may be unclassified if they do not meet the criteria. Binary images are images whose pixels have only two possible intensity values. They are often produced by applying thresholds to grayscale or color images in order to separate an object in the image from the background. The color of the object is referred to as the foreground color [12].

\section{RESULT AND DISCUSSION}

Firstly, the hyperspectral bands have been analyzed for the radiometric quality. It was revealed 
that the water absorption bands and some other bands of the image had zero values. When these bands have been excluded, the original dataset was reduced from 242 bands to 173 bands. Then, Hyperion channels were geometrically corrected to a UTM map projection using a topographic map of the study area, scale 1:100,000. The ground control point have been selected on clearly delineated areas around the Lake Khuvsgul and other clear sites. In total 12 points were selected. For the transformation, a second order transformation and nearest neighbor resampling approach have been applied and the related root mean square error was 0.78 pixel.

To define the regions for the training signature selection from the hyperspectral image, two to three areas of interest (AOI) representing the selected 6 classes have been selected. The separability of the training signatures was firstly checked in feature space and then evaluated using transformed divergence. After the investigation, the samples that demonstrated the greatest separability were chosen to form the final signatures and they included about $180-568$ pixels.

For the actual classification, spectral angle mapper, support vector machine, spectral information divergence and binary spectral encoding methods have been used.

To increase the quality of the classification results to the initially classified images, a fuzzy convolution with a $3 \times 3$ size window was applied. This technique creates a thematic layer by calculating the total weighted inverse distance of all the classes in a determined window of pixels and assigning the centre pixel the class with the largest total inverse distance summed over the entire set of fuzzy classification layers. In that case, classes with a very small distance value will remain unchanged while the classes with higher distance values might change to a neighboring value if there are a sufficient number of neighboring pixels with class values and small corresponding distance values [13]. The visual inspection of the fuzzy convolved images indicated that there are some improvements on the borders of the neighboring classes that significantly influence the separation of the decision boundaries in multidimensional feature space.

The final classified images are shown in Figure 2 (a-d). As seen from the Figure 2, the classification result of the binary spectral encoding gives the worst result, because there are high overlaps among different classes. However, these overlaps decrease or disappear on other advanced classification results. a)

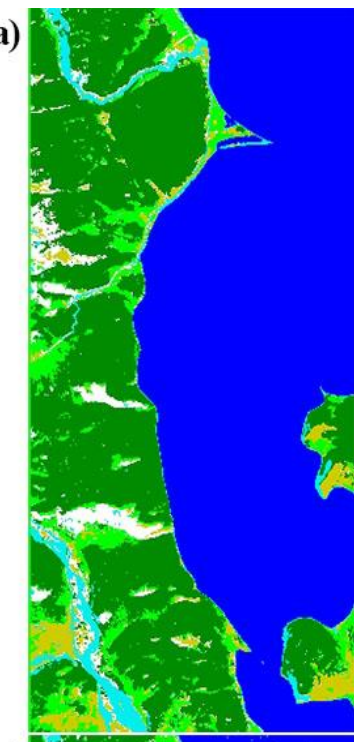

c)
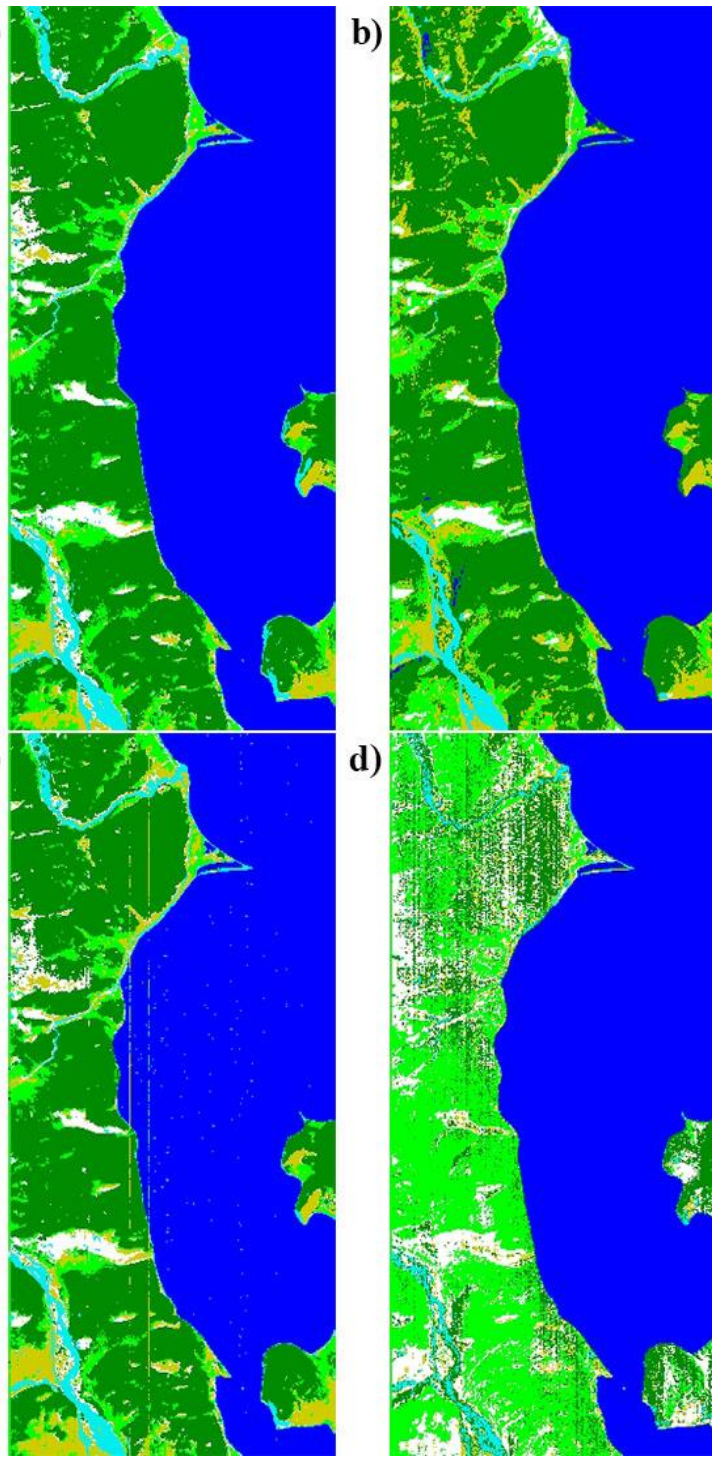

Figure 2. Classification results: a) spectral angle mapper, b) support vector machine, c) spectral information divergence, d) binary spectral encoding.

For the accuracy assessment of the classification results, the overall performance has been used. This approach creates a confusion matrix in which reference pixels are compared with the classified pixels and as a result an accuracy report is generated indicating the percentages of the overall accuracy [14].

As ground truth information, different AOIs containing 7735 purest pixels have been selected. AOIs were selected on a principle that more pixels to be selected for the evaluation of the larger classes such as coniferous forest and water than the smaller classes such as dry river bed and soil. The overall classification accuracies for the selected classes were $96.43 \%, 93.40 \%, 91.88 \%$ and $78.01 \%$ for the spectral 
angle mapper, support vector machine, spectral information divergence and binary spectral encoding, respectively.

\section{CONCLUSIONS}

The aim of this research was to classify diverse land cover types using some advanced methods applied for the hyperspectral image classification. Test site was selected in the west of the Lake Khuvsgul representing 6 main classes as coniferous forest, grassland, soil, bare land, dry river bed and water. As data sources, hyperspectral Hyperion image as well as topographic and forest taxonomy maps were available. For the classification, 4 different techniques, namely, spectral angle mapper, support vector machine, spectral information divergence and binary spectral encoding methods were applied.

When the classification results were compared, the output of the spectral angle mapper was superior to the other methods. Overall, the study showed that the current advanced hyperspectral data analysis techniques could be effectively used for the land cover discrimination.

\section{REFERENCES}

[1] Li, X., Ruolan Li, Mengyu Wang, Yaru Liu, Baohua Zhang and Jun Zhou, 2017, Hyperspectral Imaging and Their Applications in the Nondestructive Quality Assessment of Fruits and Vegetables, Hyperspectral Imaging in Agriculture, Food and Environment, IntechOpen, DOI: https://doi.org/10.5772/intechopen.72250

[2] Amarsaikhan, D., Ganzorig, M., Saandar, M., Blotevogel, H.H., Egshiglen, E., Gantuya, R., Nergui, B. and Enkhjargal, D., 2012, Comparison of multisource image fusion methods and land cover classification, International Journal of Remote Sensing, Vol.33(8), pp.2532-2550. DOI: https://doi.org/10.1080/01431161.2011.616552

[3] Camps-Valls, G. and Bruzzone, L., 2005, Kernel-based methods for hyperspectral image classification, IEEE Trans. Geosci. Remote Sens., vol. 43, pp.1351 -1362. DOI: https://doi.org/10.1109/TGRS.2005.846154

[4] Ding, H, Luming $\mathrm{Xu}$, Yue $\mathrm{Wu}$ and Wenzhong Shi, 2020, Classification of hyperspectral images by deep learning of spectral-spatial features, Arabian Journal of Geosciences volume 13,
Article number: $464 . \quad$ DOI: https://doi.org/10.1007/s12517-020-05487-4

[5] Gao, F., Qun Wang, Junyu Dong and Qizhi Xu, 2018, Spectral and spatial classification of hyperspectral images based on random multigraphs remote sensing, 10, 1271; DOI: https://doi.org/10.3390/rs10081271

[6] Batsukh, N., 2004, Forest ecosystems in Mongolia, Available at: http://d2ouvy59p0dg6k.cloudfront.net/download sarch04.pdf.

[7] EO-1, 2017, EO1 Trajectory Details, National Space Science Data Center of NASA, Available at: https://nssdc.gsfc.nasa.gov/

[8] Du, H., Chang, C.-I. and Jensen, J., 2004, New hyperspectral discrimination measure for spectral characterization, Optical Engineering, Vol. 43, No. $\quad 8, \quad 1777-1786$ DOI: https://doi.org/10.1117/1.1766301

[9] Trevor, H., Robert, T. and Jerome, F., 2008, The elements of statistical learning: data mining, inference, and prediction, Second edition, New York, Springer, pp.134.

[10]Zhang, E., Zhang, X., Yang, S. and Wang, S., 2014, Improving Hyperspectral Image Classification Using Spectral Information Divergence," in IEEE Geoscience and Remote Sensing Letters, vol. 11, no. 1, pp.249-253, DOI: https://doi.org/10.1109/LGRS.2013.2255097

[11] Khaleghi, M., Ranjbar, H., Shahabpour, J., 2014, Spectral angle mapping, spectral information divergence, and principal component analysis of the ASTER SWIR data for exploration of porphyry copper mineralization in the Sarduiyeh area, Kerman province, Iran. Appl Geomat 6, 49-58. DOI: https://doi.org/10.1007/s12518-0140125-0

[12] Xie, H. \& Tong, X., 2012, An inproved binary encoding algorithm for classification of hyperspectral images, 2012 4th Workshop on Hyperspectral Image and Signal Processing: Evolution in Remote Sensing, pp.1-4, DOI: https://doi.org/10.1109/WHISPERS.2012.68743 31

[13]ERDAS, 2009, ERDAS Field Guide, Atlanta, Georgia.

[14] Richards, J.A. and Jia, S., 2014, Remote Sensing Digital Image Analysis- An Introduction, 3rd edn (Berlin: Springer-Verlag). 